\title{
Capturing the experiences of patients with inherited optic neuropathies: a systematic review of patient-reported outcome measures (PROMs) and qualitative studies
}

\author{
Benson S. Chen ${ }^{1,2} \cdot$ Tomasz Galus $^{1} \cdot$ Stephanie Archer ${ }^{3} \cdot$ Valerija Tadić $^{4} \cdot$ Mike Horton $^{5} \cdot$ Konrad Pesudovs $^{6}$. \\ Tasanee Braithwaite $^{7,8} \cdot$ Patrick Yu-Wai-Man ${ }^{1,2,9,10}$
}

Received: 27 September 2021 / Revised: 7 December 2021 / Accepted: 16 December 2021 / Published online: 13 January 2022

(c) The Author(s) 2022

\begin{abstract}
Purpose To identify and comprehensively evaluate studies capturing the experience of individuals affected by an inherited optic neuropathy (ION), focusing on patient-reported outcome measures (PROMs) and qualitative studies where the health status and quality of life (QoL) of these individuals have been explored.

Methods Systematic review of five databases using a search strategy combining four concepts: (1) ION; (2) QoL and health status; (3) PROMs; and (4) qualitative research. Studies assessing the impact of ION on any QoL domain using a PROM or qualitative methodology were included and appraised, using criteria based on the COSMIN checklist (for PROM studies) and the CASP checklist (for qualitative studies).

Results Of 1326 unique articles identified, six studies were included. Five PROMs were identified: Visual Function Index (VF-14); Hospital Anxiety and Depression Scale (HADS); a novel graphical online assessment tool (NGOAT) for reporting emotional response to vision loss; a new PROM informed by the DSM-V Criteria for Major Depressive Disorder; and an interpersonal and career 'impact rating' PROM. The psychometric performance of included PROMs were poorly described. Qualitative studies found that vision loss resulted in psychosocial losses including loss of social and communication skills and loss of independence and freedom. Factors that modified the response to vision loss were also identified.

Conclusion The current PROMs used by individuals with ION have poor content coverage, primarily measuring activity limitation and emotional well-being, and insufficient reporting of psychometric performance. There is a need to develop a PROM for individuals ION to report their experiences of living with their condition.
\end{abstract}

\section{Key messages}

- Patient-reported outcome measures (PROMs), questionnaires that enable patients to report their experiences, are frequently used in ophthalmic clinical trials

- Current PROMs used by individuals with inherited optic neuropathies (ION) have poor content coverage, primarily focusing on vision-related activity limitation

- The experience of living with an ION includes psychosocial losses, including loss of social and communication skills and loss of independence and freedom

- Clinicians and researchers utilising PROMs with individuals affected by an ION should be aware of the limitations of pre-existing PROMs

Keywords Leber hereditary optic neuropathy · Dominant optic atrophy · Quality of life · Patient-reported outcome measure

Extended author information available on the last page of the article 


\section{Introduction}

Inherited optic neuropathies (IONs) constitute a genetically and clinically heterogeneous group of rare disorders that result in progressive optic nerve degeneration and irreversible visual loss [1,2]. Autosomal dominant optic atrophy (DOA) and Leber hereditary optic neuropathy (LHON) are the two most common IONs encountered in clinical practice, with an estimated population prevalence of 4-10 per 100,000 individuals and $2-3$ per 100,000 individuals, respectively [2]. The pathological hallmark of IONs is the preferential loss of retinal ganglion cells, which results in a central pattern of visual loss. Individuals with DOA usually become symptomatic in early childhood, whereas LHON typically presents between the ages of 15 and 35 years old with rapidly progressive visual loss. The visual prognosis is poor with the majority of individuals eventually fulfilling the legal requirement for blind registration [2]. There are currently no approved diseasemodifying treatments available to halt or reverse the loss of retinal ganglion cells. Treatment of IONs is focused on symptom management, genetic counselling, and supporting affected individuals to adapt to low vision through the provision of vision aids and occupational therapy.

In the last 10 years, there has been significant interest in the treatment of inherited ocular disorders by gene therapy, including IONs. Several Phase II and III clinical trials evaluating the effectiveness and safety of gene therapy in individuals who harbour the $\mathrm{m} .11778 \mathrm{G}>\mathrm{A}$ mutation responsible for the majority of cases of LHON have recently reached completion. Early results are promising, with a favourable safety profile and a meaningful improvement in visual acuity for study participants. If successful, gene therapy could be a reality for other IONs. Clinical trials for LHON, like other ophthalmic studies, utilise traditional metrics of visual function as study outcome measures, including visual acuity, visual fields, contrast sensitivity, and colour vision [3, 4]. Specific study endpoints vary by study design, but generally involve demonstrating a statistically significant between-group difference for the measure of interest. Assessment of best-corrected visual acuity using the Early Treatment Diabetic Retinopathy Study (ETDRS) chart remains the perceived gold standard for assessing visual function. A three-line (15 letter) change on the ETDRS chart is considered to be clinically significant and an acceptable primary endpoint for clinical trials per the US Food and Drug Administration (FDA).

Although visual acuity remains the most established parameter used by regulatory agencies such as the FDA when considering therapeutic efficacy, it is now recognised that this metric has its limitations as it does not capture the full extent of an individual's lived experience of their condition. There is increasing awareness of the importance of the patient voice in ophthalmology, with a move away from the sole use of traditional metrics of visual function in clinical trials toward inclusion of metrics that matter more to patients [5]. The patient's perspective of their health status and their experience of illness, disability, and treatment can be assessed using questionnaires known as patient-reported outcomes measures (PROMs). There has been a proliferation of PROMs developed to assess the experience of ophthalmic disorders and their treatments, particularly for use in therapeutic clinical trials, where impact on quality of life (QoL) is a frequently desired outcome measure [5-7]. In order for PROMs to be effective in clinical practice and research, they have to capture the disease characteristics that matter to the patient [5]. Generic PROMs, including generic QoL PROMs may be useful when comparing the health status or views of individuals across different disease groups. However, they may exclude important aspects that are relevant to specific groups of patients, which are better assessed using a condition-specific PROM.

It is unclear if any currently available PROMs are suitable for use in assessing the experience of individuals affected by an ION. The purpose of this systematic review was to identify and comprehensively evaluate studies capturing the experience of adults and children affected by an ION, focusing on PROMs and qualitative studies where the health status and QoL of these individuals have been explored. Specific aims of the review were to: (1) identify all studies quantitatively assessing QoL in individuals with ION, using a PROM instrument; (2) assess the quality of the identified PROM instruments by determining robustness of content development, psychometric properties including validity and reliability, and responsiveness; (3) identify and appraise qualitative studies of individuals with ION; and (4) identify key themes and messages of the experience of individuals with ION in qualitative studies.

\section{Method}

A systematic electronic database search was undertaken using MEDLINE, EMBASE, PsycINFO, CINAHL Plus, and Scopus. The search strategy utilised index (MeSH) terms and free text words and included four concepts: (1) inherited optic neuropathies; (2) quality of life and health status; (3) patient-reported outcome measures; and (4) qualitative research. The search strategy was adapted for use depending on the database [Appendix 1]. A manual search of the reference lists and citations of included papers for articles that may have been missed by the initial electronic database search was also performed. The search strategy did not 
Table 1 Study inclusion and exclusion criteria

Inclusion Criteria:

- studies that quantitatively assessed the impact of IONs on any domain of QoL

- studies that reported on the development, psychometric assessment, or validation of

PROMs to assess the impact of IONs on any domain of QoL

- studies that qualitatively explored the impact of IONs on any domain of QoL,

through the use of focus groups, structured/semi-structured interviews or question-

naires, and/or literature reviews
Exclusion Criteria:

- animal studies and experimental science studies

- single case reports, editorials, and conference abstracts

IONs inherited optic neuropathies; PROM patient-reported outcome measure; $Q o L$ quality of life

incorporate any date or language limits. Inclusion and exclusion criteria are summarised in Table 1 .

Search results were uploaded to Endnote X8 (Thomson Reuters). After removal of duplicates, titles and abstracts of studies retrieved using the search strategy and those from additional sources were screened by one reviewer (BC) to remove irrelevant articles. A second reviewer (SA) crosschecked $300(22 \%)$ of the studies, and Cohen's Kappa was calculated to determine inter-rater reliability of the two reviewers. Full text articles were obtained for studies that met the inclusion criteria. Abstracts that did not provide sufficient information to make a decision were taken forward for full-text screening, to minimise the risk of missing a potentially relevant article. Two reviewers (BC and TG) then independently performed a full-text screen. Reasons for exclusion at the full-text stage of screening were recorded. A third reviewer (KP) was consulted if there was disagreement between the two reviewers. The two reviewers independently extracted data from studies meeting the inclusion criteria, using a standardised form.

For each included study, study characteristics (publication year, citation, country/region, sample size, PROM instrument(s)) and characteristics of patients for whom the instrument was developed/assessed/validated, were extracted. This included the type(s) of IONs. For qualitative studies, any identified themes and direct patient quotes/extracts were extracted. The psychometric quality of each identified PROM instrument was assessed using previously described quality criteria [8], based on the FDA guidance document and the 'COnsensus-based Standards for the selection of health Measurement INstruments' (COSMIN) risk of bias assessment tool [Appendix 2]. The quality of qualitative studies was assessed using the 'Critical Appraisal Skills Programme' (CASP) checklist for qualitative studies [9].

Extracted data from both quantitative and qualitative studies was compiled in a database and then presented separately in tabular form. The characteristics of the patient groups studied (sample size, age, sex, ethnicity, type of ION, comorbidities, treatments) in both types of studies were summarised. For the data extracted from quantitative studies, the PROM instrument names, QoL domains assessed, and the number of questions included for each domain within the instrument were summarised. For each PROM instrument, the quality assessment for each criterion was summarised and a narrative summary of the findings was provided. For qualitative studies, the methodological approach, participant information and inclusion criteria, study location, and summary of the main findings were reported in tabular format. Subgroup analysis based on individual QoL domains was also conducted, by presenting PROMs by QoL domain or construct, and by content coverage, in tabular format.

The protocol was registered with PROSPERO (CRD42020221807).

\section{Results}

Figure 1 depicts the flow of information through the different phases of the systematic review. The search conducted on December 1, 2020 identified a total of 1897 studies from MEDLINE (589), EMBASE (1085), PsycINFO (76), CINAHL Plus (16), and Scopus (131). After removal of 571 duplicates, the titles and abstracts of 1,326 studies were screened for eligibility. A sample of 300 studies was cross-checked by the second reviewer, with excellent agreement (99.33\% agreement; Cohen's к: 0.83 ) between the two reviewers. One-hundred and seventy-four studies underwent full-text screen. After excluding 168 studies, a total of six studies were included in the review. A manual search of the reference lists of included studies did not yield any additional studies that met eligibility criteria.

\section{Characteristics of identified studies and study participants}

The characteristics of the six included studies are summarised in Table 2 [10-15]. Five studies reported the experiences of individuals with LHON [11-15], and one study focused on the experiences of participants with DOA and 'DOA +' (a clinical syndrome comprising of DOA with extra-ocular manifestations) [10]. One study from a group in the USA involved the development of an online assessment tools for individuals with LHON to retrospectively report and track the emotional impact of their condition using a mixed methods approach [13]. The same group also determined the prevalence of depressive symptoms 


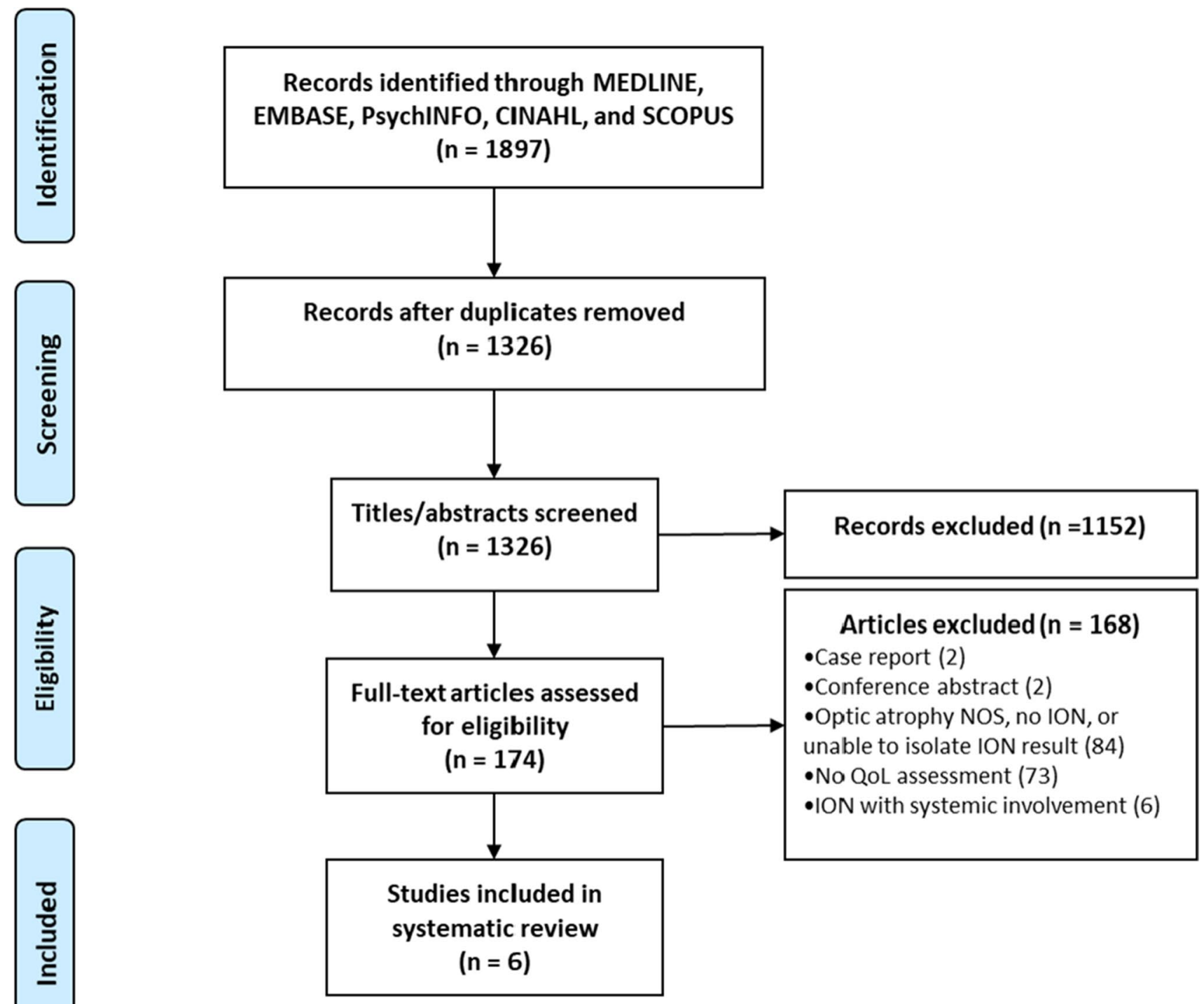

Fig. 1 Systematic review flow diagram. ION: inherited optic neuropathy; NOS: not otherwise specified; QoL: quality of life

in individuals with LHON and the impact of their condition on interpersonal relationships and careers, using two self-reported measures in a second study [14]. Two studies reported the vision-related QoL of individuals with LHON and unaffected carriers $[11,15]$. One study utilised qualitative methods to describe the lived experiences of seven men affected by LHON living in the UK [12]. The single study involving individuals with DOA and DOA + described vision-related QoL and the prevalence of mood symptoms [10]. The findings of the six included studies are summarised in Appendix 3.

Five hundred and two participants, across four of the identified studies, were recruited from the UK, the Netherlands, Germany, and China [10-12, 15]. The two US studies recruited 219 participants internationally through social media and the mailing lists of LHON patient advocacy groups $[13,14]$. A breakdown of the location of participants was not provided in these two studies. Consistent with the male predominance of affected individuals, most participants $(n=430)$ in the five studies of individuals with LHON were male. Except for one study of $55 \mathrm{Chi}-$ nese individuals affected by LHON [11], the majority of participants $(n=534)$ were White. The average age of participants was between 30 and 50 years, although a range of ages was represented. The duration of disease was not uniformly reported in all studies, but included recently diagnosed individuals and those with chronic disease. Three studies reported the genetic mutation of participants $[10,11,15]$. 


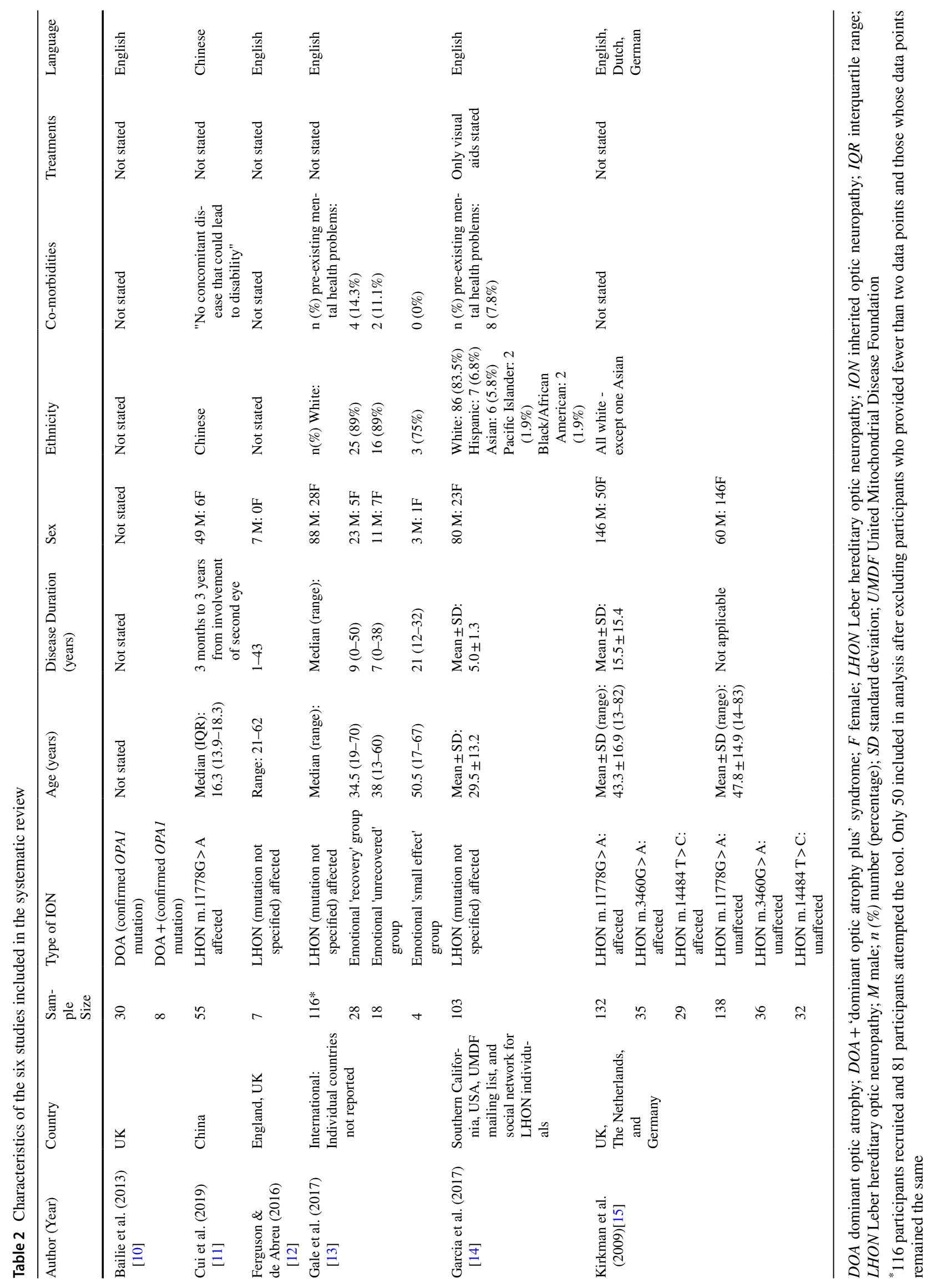




\section{Identified PROMS and quality assessment}

A total of five PROMs used with individuals with ION were identified (Table 3). The Visual Function Index (VF-14), an instrument that measures vision-related activity limitation, was used by three studies to report vision-related QoL $[10,11,15]$. Three instruments were used as measures of emotional wellbeing: the Hospital Anxiety and Depression Scale (HADS); a 'novel graphical online assessment tool' (NGOAT); and a measure informed by the Diagnostic and Statistical Manual of Mental Disorders V (DSM-V) criteria for Major Depressive Disorder (MDD). The HADS, a 14-item scale comprised of a 7-item anxiety domain and a 7-item depression domain, was used in the single study of individuals with DOA "to quantify the functional impact of DOA on patients' QoL" [10]. The NGOAT is a single item instrument developed to allow participants with LHON to retrospectively report their emotional response to vision loss on a 10-point rating scale [13]. The DSM-V criteria for MDD [16], was used to guide the domains of a 9-item measure for assessing the psychological well-being of individuals with LHON [14]. The impact of disease on social wellbeing and work/productive activity was assessed in the same study of individuals with LHON using a 1-item interpersonal interactions and 1-item career goals 'impact rating' on a 21-point scale [14].

None of the included studies provided sufficient information to enable comprehensive assessment of the psychometric quality of the five identified PROMs (Table 3). The three studies utilising pre-existing PROMs (VF-14 and HADS) did not justify the selection of the instruments used [10, $11,15]$. The performance of the VF-14 was compared to the visual acuity of participants in two studies (convergent validity) $[10,11]$. One study found a moderate correlation between $\log$ MAR visual acuity and VF-14 scores, that is, that as visual acuity worsened in individuals with DOA or $\mathrm{DOA}+$, the lower their VF-14 scores (indicating worsening vision-related activity limitation) [10]. The same study also found a moderate correlation between HADS and VF-14 scores. The study involving Chinese individuals with LHON found an inconsistent correlation between VF-14 scores and visual acuity at different time points, but did not report a correlation co-efficient [11]. The same study detected clinically important changes in VF-14 scores over their study period (responsiveness). Other instrument properties such as discriminant validity, predictive validity, and test-retest reliability could not be determined.

The two studies that described the development of the NGOAT [13], and utilised the DSM-V criteria for MDD [14] and 'impact rating' PROMs [14], provided a clear statement of the aims of the outcome measures. The items appeared relevant to the intended population (face validity). However, individuals with ION were not involved in identifying or selecting the items. The authors of the NGOAT study also acknowledged that the ambiguous wording of the single item in their PROM, "to what extent have you felt sad?", invalidated any 'advanced' quantitative analysis of the data they had planned. Instead, the pattern of change in NGOAT scores before, during, and after vision loss was qualitatively analysed with the comments that participants provided. The instrument appeared to have some responsiveness to change, but the data did not allow measurement of a minimally important difference. There was no justification for the response categories used, including the 10-point scale for the NGOAT or 21-point scale for the 'impact rating' PROMs. The 'impact rating' PROMs also showed significant skew and floor effects. The psychometric performance of the NGOAT, the PROM based on the DSM-V criteria for MDD, and the 'impact rating' PROMs, were not reported and could not be determined from the information provided.

\section{Qualitative studies and quality assessment}

The development of the NGOAT to retrospectively assess emotional response to vision loss in individuals with LHON used a mixed methods approach [13]. Participants were asked to justify the rating scores they had indicated on the scale. These responses were analysed qualitatively, focusing on factors that affected mood and adjustment, and recovery for participants. These were further analysed for themes and patterns. However, the qualitative methodology was not discussed and there was no acknowledgement of the reflexivity of the researchers when analysing the collected data. The study found that the extent and duration of sadness was considerably worse when vision loss resulted in loss of relationships, independence, and work or college (university) training. Recovery of mood was also related to these factors; with retraining, new employment, career development, financial security, development of a relationship and marriage, having children, visual aid technology, and sports and travels, all cited as factors associated with recovery of mood. Vulnerability factors in the form of severe hardships such as bereavement, drug or alcohol misuse, academic underachievement, and unplanned parenthood, were observed in participants with long periods of sadness or no recovery of mood after vision loss.

The single qualitative study identified in the review used semi-structured interviews informed by interpretative phenomenological analysis principles to examine the lived experiences of seven men affected by LHON in the UK [12]. One of the study authors had LHON himself and recognised the effect this could have in collecting and analysing the data. This was acknowledged in the study methodology. The study identified six key themes: (1) loss of vision resulted in psychosocial losses including loss of social/communication skills, loss of independence and freedom; (2) participants 
demonstrated a determination and resolve that enabled them to continue with their lives and overcome challenges; (3) practical methods to adjust to life without vision required developing new skills and using other sensory tools to obtain information; (4) the level of residual vision that remained post LHON-onset appeared to influence participants' decision to display their blind identity; (5) clarity of a participant's visual field influenced how they viewed regaining their independence; and (6) all participants acknowledged they experienced moments that evoked a feeling of loss of their sight. Using the CASP checklist, the overall quality of the identified study was excellent (Appendix 4). The only potential source of bias was the study recruitment strategy, which involved advertising the study through two social networking websites and by directly emailing individuals registered with an LHON website. The study authors recognised that it would be erroneous to generalise the results of the study across the LHON population because of the participants that were recruited [12].

\section{Discussion}

There is a paucity of studies reporting the experiences of individuals with ION. This systematic review identified six studies capturing the experiences of individuals with ION, using five PROMs: two pre-existing PROMs not developed for use with individuals with ION per se (VF-14 and HADS) and three new measures for use by individuals affected by LHON (NGOAT for assessing emotional response to vision loss, a measure based on the DSM-V criteria for MDD, and an interpersonal interactions and career goals 'impact rating'). The studies found that individuals with ION experienced limitations in their activity as a consequence of their vision loss. Many participants also reported symptoms of anxiety and depression, with some individuals affected by LHON satisfying DSM-V criteria for major depression. There was also considerable impact on interpersonal relationships and career.

In research involving individuals with ophthalmic conditions, PROMs should be comprehensive to assess a holistic impact in QoL and include content that is important to the patient group utilising the PROM. Quality of life is a broad, multi-dimensional concept defined by the World Health Organisation as "an individual's perception of their position in life in the context of the culture and value systems in which they live and in relation to their goals, expectations, standards and concerns" [17]. In ophthalmology, a set of ten QoL domains have been identified previously as being important to patients with ophthalmic conditions including: activity limitation, mobility, visual symptoms, ocular surface symptoms, general symptoms, emotional well-being, social participation, economic, health concerns, and convenience $[18,19]$. It is unclear if these domains are relevant or important to individuals with ION. Ocular surface symptoms, for example, are not a feature of IONs, but much more common in diseases such as dry eye syndrome or blepharitis. Despite QoL being a key concept of interest in three of the studies identified in the systematic review, the PROMs that were selected or developed by the researchers primarily focused on activity limitation and emotional well-being. Individuals with ION were not involved in the selection or design of the PROMs identified.

In the literature, constructs like vision-related activity limitation and vision-related QoL are frequently merged with visual function when discussing the patient experience of having an ophthalmic disorder [7, 17], resulting in the selection of PROMs that may not be appropriate for the concept of interest. The VF-14, which was used as a measure of vision-related QoL in three studies [10,11,15], was originally designed for patients undergoing cataract surgery and is a widely-used and validated tool that quantifies visionrelated activity limitation [20]. Despite this, the VF-14 has been used in clinical trials as a measure for vision-related QoL, including a therapeutic clinical trial for idebenone in individuals with LHON, which did not find a statistically significant difference between the active treatment and placebo groups using the VF-14 [21]. Similar issues have been identified in ophthalmic disease-specific PROMs. A systematic review in 2017 of retina-specific PROMs for retinal diseases such as age-related macular degeneration and diabetic retinopathy, found that existing retina-specific PROMs primarily measured activity limitation or mobility [6].

Factors such as the age and demographic background of the individuals using the PROM also need to be taken into consideration when selecting or developing a PROM. Items in a PROM must be relevant to the group that is utilising the PROM. Although the VF-14 shows high internal consistency and is a reliable, valid instrument for measuring vision-related activity limitation, several items such as difficulty "doing fine handwork like sewing, knitting, crocheting and carpentry" and difficulty "playing games such as bingo, dominos, card games or mahjong" are more relevant to older individuals with cataracts, than young individuals affected by an ION [20]. Similar issues may also occur when using PROMs developed for individuals with low vision. PROMs such as the Veterans Affairs Low-Vision Visual Functioning Questionnaire (VF-VFQ-48) and the Impact of Vision Impairment Profile (IVI), are often developed with the input of individuals affected by glaucoma and age-related macular degeneration, both age-related disorders which are more prevalent in the older population [22, 23]. Individuals affected by an ION are likely to have different needs to those of older patients with low vision.

Impact of LHON on interpersonal relationships and career was measured in one study [14], but only a single item was used to assess the impact of LHON in each of these social participation domains. The majority of participants 


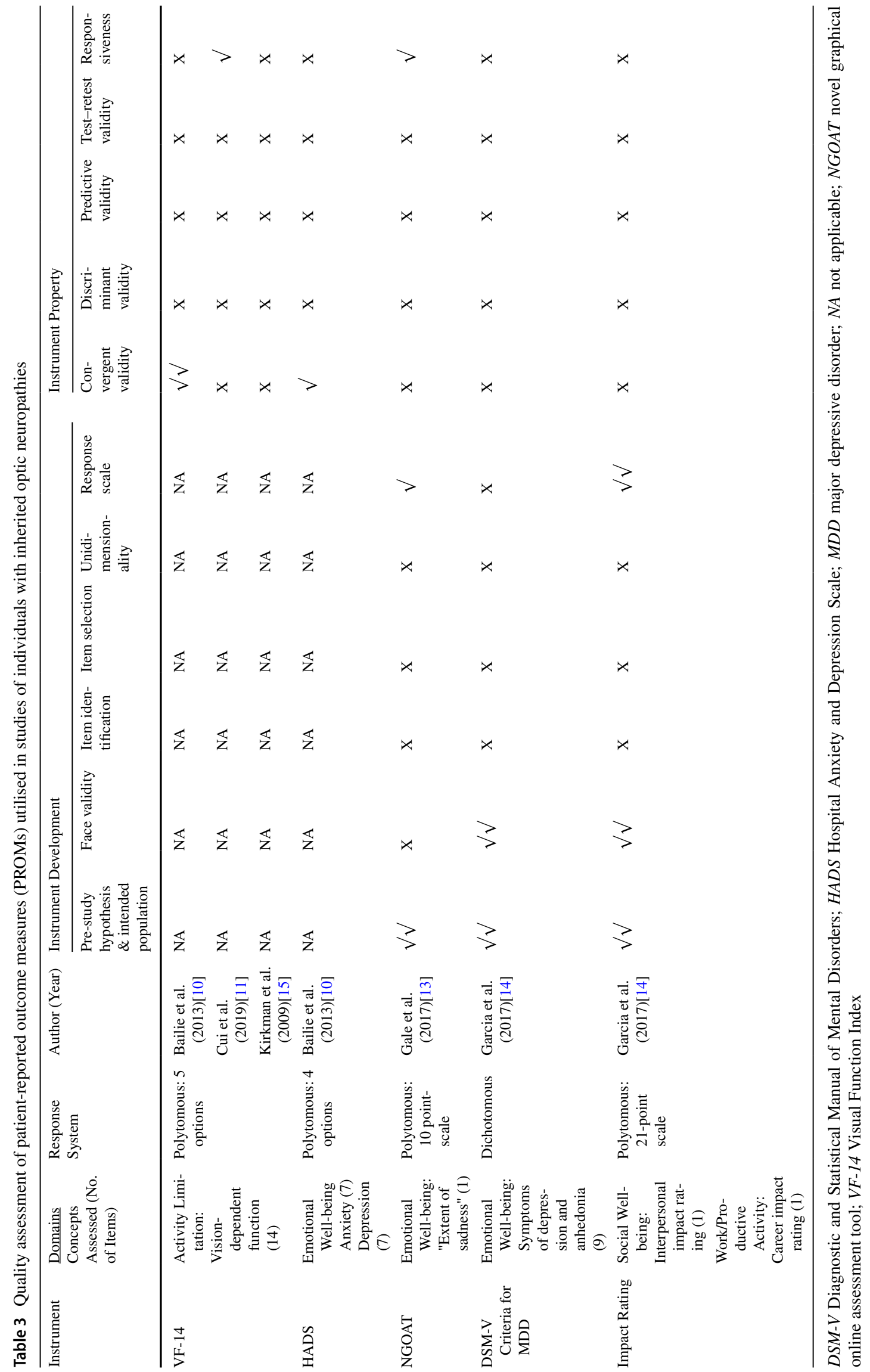


in this study indicated the lowest possible score (indicating a negative impact). In contrast, the studies that used a qualitative or mixed methods approach, obtained rich detail regarding the impact of LHON on interpersonal relationships and career/education, and the factors that impacted on their emotional response to vision loss. Importantly, both studies identified factors that influenced how individuals with ION responded to these challenges, including diseaserelated factors, personal factors and attitudes, and external/ environmental factors. These factors are consistent with those identified by Albrecht and Devlieger [24], in their 'balance theory' explanation for the disability paradox, the observation that many individuals with serious and persistent disabilities actually report that they experience a good or excellent QoL, despite their poor health status or disability. They theorised that the experience of well-being and life satisfaction (good QoL) was contingent on achieving balance between body, mind, and spirit in the context of the larger environment. Factors contributing to good QoL included a 'can-do' approach to life and belief that one still had control over their bodies, minds, and lives. For some individuals with disability that they interviewed, disability served to clarify and re-orient their lives. Factors contributing to poor QoL included pain, fatigue, a sense of hopelessness, loss of control of corporal or mental activities, and/or not having a clear purpose or spiritual outlook in life [24].

A key strength of the systematic review was its comprehensive design, which involved multiple databases including those focused on behavioural and social science research and literature, and the inclusion of studies utilising qualitative methodology to gain a more holistic understanding of the experiences of individuals affected by an ION. Despite this, very few studies were identified. Studies, particularly clinical trials, involving individuals with rare disease are often not completed or published [25]. Several reasons for this include lack of funding, difficulty recruiting sufficient patient numbers, and the challenges associated with publishing studies with negative results. Despite the importance of qualitative research addressing concepts that are difficult to quantify numerically such as QoL, feelings, or opinions, only one study utilising qualitative methods was identified in the systematic review. Qualitative research is significantly under-represented in ophthalmology journals. A study in 2017 , found that despite $11 \%$ of studies identified on the UK Clinical Research Network portfolio utilising qualitative research methods, $0.3 \%$ of original research published in three major ophthalmology journals included qualitative methods during the time period screened [26]. Another limitation is the predominance of white men participating in the included studies, which impacts on the generalisability of the studies' findings. Further qualitative studies are vital to understand the experience of living with an ION, and could inform the content development of an ION-specific
PROM. Although individuals with DOA and LHON share similar demographic features such as age of onset, studies identifying similarities and differences in the experiences of individuals with both conditions are required. Given the challenges of developing PROMs for rare diseases, there may be an opportunity to develop a PROM that can also be utilised by individuals with inherited retinal disorders that share similar clinical features, including pattern of visual field impairment.

In conclusion, this systematic review found that the PROMs currently used to report the experiences of individuals affected by an ION are limited in content coverage. This arises from problematic selection of pre-existing PROMs or screening tools that are not intended as measures of QoL for this population, and problems with the content development of new measures. Relying on PROMs such as the VF-14 as a clinical trial endpoint for reporting QoL is highly problematic as the items do not comprehensively cover the QoL domains that are important to individuals with ION. Additionally, the psychometric properties of the identified PROMs were not uniformly reported, calling into question the validity of these instruments for use with the ION population. There is a need to develop an ION-specific PROM for individuals with DOA and LHON to report their experiences of living with their condition, that can be used in future clinical trials and natural history studies. The psychometric properties of the PROM also need to be evaluated in the development of the PROM, to ensure that the findings are robust when used in studies involving individuals with ION.

Supplementary Information The online version contains supplementary material available at https://doi.org/10.1007/s00417-021-05534-0.

Author contribution All authors contributed to the study conception and design. Material preparation, data collection and analysis were performed by BSC, TG and SA. The first draft of the manuscript was written by BSC. All authors read and approved the final manuscript.

Data availability The authors confirm that the data supporting the findings of this study are available within the article and its supplementary materials.

Code availability Not applicable.

\section{Declarations}

Conflict of interest BSC is recipient of the Cambridge-Rutherford Memorial Scholarship awarded by the Royal Society Te Apārangi - Rutherford Foundation and the Cambridge Commonwealth, European \& International Trust, and also the Aotearoa New Zealand Fellows Research Entry Scholarship awarded by the Royal Australasian College of Physicians (RACP). PYWM is supported by an Advanced Fellowship Award (NIHR301696) from the UK National Institute of Health Research (NIHR) and a Clinician Scientist Fellowship Award (G1002570) from the UK Medical Research Council (MRC). PYWM also receives funding from Fight for Sight (UK), the Isaac Newton Trust (UK), Moorfields Eye Charity (GR001376), the Addenbrooke's 
Charitable Trust, the National Eye Research Centre (UK), the International Foundation for Optic Nerve Disease (IFOND), the NIHR as part of the Rare Diseases Translational Research Collaboration, the NIHR Cambridge Biomedical Research Centre (BRC-1215-20014), and the NIHR Biomedical Research Centre based at Moorfields Eye Hospital NHS Foundation Trust and UCL Institute of Ophthalmology. The views expressed are those of the author(s) and not necessarily those of the NHS, the NIHR or the Department of Health.

Open Access This article is licensed under a Creative Commons Attribution 4.0 International License, which permits use, sharing, adaptation, distribution and reproduction in any medium or format, as long as you give appropriate credit to the original author(s) and the source, provide a link to the Creative Commons licence, and indicate if changes were made. The images or other third party material in this article are included in the article's Creative Commons licence, unless indicated otherwise in a credit line to the material. If material is not included in the article's Creative Commons licence and your intended use is not permitted by statutory regulation or exceeds the permitted use, you will need to obtain permission directly from the copyright holder. To view a copy of this licence, visit http://creativecommons.org/licenses/by/4.0/.

\section{References}

1. Newman NJ, Biousse V (2004) Hereditary optic neuropathies. Eye (Lond) 18:1144-1160. https://doi.org/10.1038/sj.eye.6701591

2. Yu-Wai-Man P, Griffiths PG, Chinnery PF (2011) Mitochondrial optic neuropathies - Disease mechanisms and therapeutic strategies. Prog Retin Eye Res 30:81-114. https://doi.org/10.1016/j. preteyeres.2010.11.002

3. Csaky KG, Richman EA, Ferris FL 3rd (2008) Report from the NEI/FDA Ophthalmic Clinical Trial Design and Endpoints Symposium. Invest Ophthalmol Vis Sci 49:479-489. https://doi.org/ 10.1167/iovs.07-1132

4. Lam BL, Feuer WJ, Schiffman JC, Porciatti V, Vandenbroucke R, Rosa PR, Gregori G, Guy J (2014) Trial end points and natural history in patients with G11778A Leber hereditary optic neuropathy : Preparation for gene therapy clinical trial. JAMA Ophthalmol 132:428-436. https://doi.org/10.1001/jamaophthalmol.2013.7971

5. Braithwaite T, Calvert M, Gray A, Pesudovs K, Denniston AK (2019) The use of patient-reported outcome research in modern ophthalmology: Impact on clinical trials and routine clinical practice. Patient Relat Outcome Meas 10:9-24. https://doi.org/ 10.2147/PROM.S162802

6. Prem Senthil M, Khadka J, Pesudovs K (2017) Assessment of patient-reported outcomes in retinal diseases: a systematic review. Surv Ophthalmol 62:546-582. https://doi.org/10.1016/j.survo phthal.2016.12.011

7. Tadic V, Hogan A, Sobti N, Knowles RL, Rahi JS (2013) Patientreported outcome measures (PROMs) in paediatric ophthalmology: A systematic review. Br J Ophthalmol 97:1369-1381. https:// doi.org/10.1136/bjophthalmol-2013-303350

8. Fernandes S, Fond G, Zendjidjian XY, Baumstarck K, Lancon C, Berna F, Schurhoff F, Aouizerate B, Henry C, Etain B, Samalin L, Leboyer M, Llorca PM, Coldefy M, Auquier P, Boyer L, French PG (2020) Measuring the patient experience of mental health care: A systematic and critical review of patient-reported experience measures. Patient Prefer Adherence 14:2147-2161. https://doi.org/10.2147/PPA.S255264

9. Critical Appraisal Skills Programme (CASP) (2018) CASP qualitative checklist. https://casp-uk.b-cdn.net/wp-content/uploads/ 2018/03/CASP-Qualitative-Checklist-2018_fillable_form.pdf. Accessed 2 December 2020
10. Bailie M, Votruba M, Griffiths PG, Chinnery PF, Yu-Wai-Man P (2013) Visual and psychological morbidity among patients with autosomal dominant optic atrophy. Acta Ophthalmol 91:e413-414. https://doi.org/10.1111/aos.12077

11. Cui S, Jiang H, Peng J, Wang J, Zhang X (2019) Evaluation of Vision-Related Quality of Life in Chinese Patients With Leber Hereditary Optic Neuropathy and the G11778A Mutation. J Neuroophthalmol 39:56-59. https://doi.org/10.1097/WNO.00000 00000000644

12. Ferguson J, de Abreu G (2016) What is the lived experience for people with Leber hereditary optic neuropathy? Br J Vis Impair 34:109-120. https://doi.org/10.1177/0264619615616260

13. Gale J, Khoshnevis M, Frousiakis SE, Karanjia R, Poincenot L, Sadun AA, Baron DA (2017) An international study of emotional response to bilateral vision loss using a novel graphical online assessment tool. Psychosomatics 58:38-45. https://doi.org/10. 1016/j.psym.2016.07.002

14. Garcia GA, Khoshnevis M, Gale J, Frousiakis SE, Hwang TJ, Poincenot L, Karanjia R, Baron D, Sadun AA (2017) Profound vision loss impairs psychological well-being in young and middleaged individuals. Clin Ophthalmol 11:417-427. https://doi.org/10. 2147/OPTH.S113414

15. Kirkman MA, Korsten A, Leonhardt M, Dimitriadis K, De Coo IF, Klopstock T, Griffiths PG, Hudson G, Chinnery PF, Yu-Wai-Man P (2009) Quality of life in patients with Leber hereditary optic neuropathy. Invest Ophthalmol Vis Sci 50:3112-3115. https://doi. org/10.1167/iovs.08-3166

16 American Psychiatric Association (2013) Diagnostic and statistical manual of mental disorders: diagnostic and statistical manual of mental disorders, 5th edn. American Psychiatric Association, Arlington

17. World Health Organization (WHO) (2001) International classification of functioning, disability and health: ICF. http://whqlibdoc. who.int/publications/2001/9241545429.pdf. Accessed 2 December 2020

18. Fenwick EK, Pesudovs K, Khadka J, Dirani M, Rees G, Wong TY, Lamoureux EL (2012) The impact of diabetic retinopathy on quality of life: qualitative findings from an item bank development project. Qual Life Res 21:1771-1782. https://doi.org/10. 1007/s11136-012-0110-1

19. Khadka J, McAlinden C, Craig JE, Fenwick EK, Lamoureux EL, Pesudovs K (2015) Identifying content for the glaucoma-specific item bank to measure quality-of-life parameters. J Glaucoma 24:12-19. https://doi.org/10.1097/IJG.0b013e318287ac11

20. Steinberg EP, Tielsch JM, Schein OD, Javitt JC, Sharkey P, Cassard SD, Legro MW, Diener-West M, Bass EB, Damiano AM et al (1994) The VF-14. An index of functional impairment in patients with cataract. Arch Ophthalmol 112:630-638. https://doi.org/10. 1001/archopht.1994.01090170074026

21. National Institute for Health and Care Excellence (NICE) Commissioning Support Programme (2020) Clinical commissioning policy: Idebenone for treating people over 12 years of age with Leber's hereditary optic neuropathy. https://www.england.nhs.uk/ wp-content/uploads/2020/07/Idebenone-for-treating-people-over12-years-of-age-with-LHO-Neuropathy.pdf. Accessed 2 December 2020

22. Stelmack JA, Szlyk JP, Stelmack TR, Demers-Turco P, Williams RT, Moran D, Massof RW (2004) Psychometric properties of the Veterans Affairs Low-Vision Visual Functioning Questionnaire. Invest Ophthalmol Vis Sci 45:3919-3928. https://doi.org/10.1167/ iovs.04-0208

23. Weih LM, Hassell JB, Keeffe J (2002) Assessment of the impact of vision impairment. Invest Ophthalmol Vis Sci 43:927-935

24. Albrecht GL, Devlieger PJ (1999) The disability paradox: High quality of life against all odds. Soc Sci Med 48:977-988. https:// doi.org/10.1016/s0277-9536(98)00411-0 
25. Rees CA, Pica N, Monuteaux MC, Bourgeois FT (2019) Noncompletion and nonpublication of trials studying rare diseases: A cross-sectional analysis. PLoS Med 16:e1002966. https://doi.org/ 10.1371/journal.pmed.1002966

26. Jones RK, Jefferis JM (2017) Is qualitative research under-represented in ophthalmology journals? Eye (Lond) 31:1117-1119. https://doi.org/10.1038/eye.2017.49
Publisher's note Springer Nature remains neutral with regard to jurisdictional claims in published maps and institutional affiliations.

\section{Authors and Affiliations}

\section{Benson S. Chen ${ }^{1,2} \cdot$ Tomasz Galus $^{1} \cdot$ Stephanie Archer ${ }^{3} \cdot$ Valerija Tadić $^{4} \cdot$ Mike Horton $^{5} \cdot$ Konrad Pesudovs $^{6}$. Tasanee Braithwaite $^{7,8} \cdot$ Patrick Yu-Wai-Man ${ }^{1,2,9,10}$}

\section{Benson S. Chen}

bsc33@cam.ac.uk

1 John Van Geest Centre for Brain Repair and MRC Mitochondrial Biology Unit, Department of Clinical Neurosciences, University of Cambridge, Cambridge, UK

2 Cambridge Eye Unit, Addenbrooke's Hospital, Cambridge University Hospitals, Cambridge, UK

3 Primary Care Unit, Department of Public Health and Primary Care, University of Cambridge, Cambridge, UK

4 School of Human Sciences, University of Greenwich, London, UK

5 Psychometric Laboratory for Health Sciences, University of Leeds, Leeds, UK
6 School of Optometry and Vision Science, University of New South Wales, Kensington, Australia

7 School of Life Course Sciences, King's College London, London, UK

8 The Medical Eye Unit, Guy's and St Thomas' NHS Foundation Trust, London, UK

9 Moorfields Eye Hospital NHS Foundation Trust, London, UK

10 Institute of Ophthalmology, University College London, London, UK 